\title{
ARTICLE \\ sFRP1 protects H9c2 cardiac myoblasts from doxorubicin- induced apoptosis by inhibiting the Wnt/PCP-JNK pathway
}

\author{
Yue-huai Hu' ${ }^{1}$, Jie Liu ${ }^{1,2}$, Jing Lu' ${ }^{1}$, Pan-xia Wang ${ }^{1}$, Jian-xing Chen ${ }^{1}$, Ying Guo ${ }^{1}$, Fang-hai Han ${ }^{3}$, Jun-jian Wang ${ }^{1}$, Wei Li ${ }^{4}$ and Pei-qing Liu ${ }^{1,5}$
}

\begin{abstract}
Doxorubicin (Dox) is an effective chemotherapy drug against a wide range of cancers, including both hematological and solid tumors. However, the serious cardiotoxic effect restricted its clinical application. We previously have illuminated the protective role of canonical Wnt/B-catenin signaling in Dox-induced cardiotoxicity. Secreted frizzled-related protein 1 (sFRP1) is one of the endogenous inhibitors of both canonical and noncanonical Wnt signaling. In this study, we investigated the relationship between sFRP1 and noncanonical Wnt/PCP-JNK (Wnt/planar cell polarity-C-Jun N-terminal kinase) pathway in Dox-induced cardiotoxicity in vitro and in vivo. We showed that treatment of $\mathrm{H} 9 \mathrm{c} 2$ cardiac myoblasts with Dox $(1 \mu \mathrm{M})$ time-dependently suppressed cell viability accompanied by significantly decreased SFRP1 protein level and increased Wnt/PCP-JNK signaling. Pretreatment with SP600125, the Wnt/PCP-JNK signaling inhibitor, attenuated Dox-induced apoptosis of H9c2 cells. Overexpression of sFRP1 protected H9c2 cells from Dox-induced apoptosis by inhibiting the Wnt/PCP-JNK pathway. After intraperitoneal injection of a cumulative dose of $15 \mathrm{mg} / \mathrm{kg}$ Dox, rats displayed significant cardiac dysfunction; their heart showed inhibited Wnt/ß-catenin signaling and activated Wnt/PCP-JNK signaling. These results suggest that SFRP1 may be a novel target for Dox-induced cardiotoxicity.
\end{abstract}

Keywords: sFRP1; doxorubicin; Wnt/PCP-JNK pathway; apoptosis; Wnt/ß-catenin signaling; SP600125

Acta Pharmacologica Sinica (2020) 41:1150-1157; https://doi.org/10.1038/s41401-020-0364-z

\section{INTRODUCTION}

Doxorubicin (Dox) is a potent antineoplastic compound with wide clinical applications in both hematological and solid tumors [1]. However, the clinical use of Dox is limited by the cardiac damage it causes [2]. Although the precise mechanisms responsible for Dox cardiotoxicity are not well explained, various mechanisms have been suggested to be involved, including reactive oxygen species production, cell death, and inflammation [3, 4]. Doxinduced apoptosis is a programmed process of death characterized by cell shrinkage, chromatin condensation, and nuclear fragmentation [3].

Wnt signaling is an evolutionarily conserved pathway that plays an important role in a diverse range of cellular activities, including cell proliferation, calcium homeostasis, and cell polarity [5]. The best understood Wnt signaling pathway is the canonical Wnt/ $\beta$ catenin pathway [6]. In our previous study, we illuminated the protective role of the $\mathrm{Wnt} / \beta$-catenin signaling pathway in Doxinduced cardiotoxicity [7]. To gain further understanding on the function of alternative Wnt signaling pathway on Dox-induced apoptosis, we focus on the noncanonical Wnt/planar cell polarity (PCP) pathway in this study. The Wnt/PCP pathway transduces signals by activating c-Jun N-terminal kinase (JNK) [8], which leads to the expression of Ptk 7, Daam 1, Vangl2, and others $[9,10]$.

The family of secreted frizzled-related proteins (sFRPs) are reportedly antagonists of Wnt signaling [11]. sFRP1, a member of the sFRP family, is highly expressed in the heart $[7,12]$ and plays an important role in many cardiac diseases by regulating Wnt signaling [13]. The results from genetic and biochemical analyses revealed that SFRP1 regulates both the canonical Wnt/ $\beta$-catenin and noncanonical Wnt/PCP-JNK pathways $[14,15]$. We explored the regulatory relationship between SFRP1 and Wnt/ $\beta$-catenin signaling in Dox-induced cardiotoxicity in our previous study. Whether the regulatory effect of sFRP1 on the Wnt/PCP-JNK pathway is involved in Dox-induced cell injury is the core issue to be resolved in this paper.

\section{MATERIALS AND METHODS}

Chemical regents

Dox (purity of 99.37\%) was purchased from Target Molecule Corp. (USA) and dissolved in sterile water to $1 \mathrm{mM}$. SP600125 (JNK inhibitor, purity of $99.00 \%$ ) was obtained from Selleck Chemicals

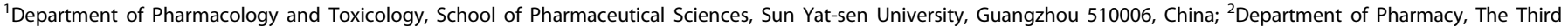

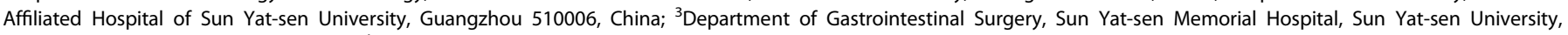

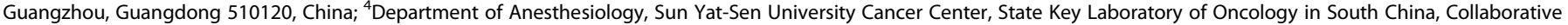

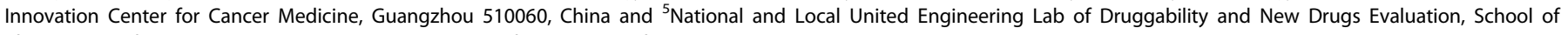
Pharmaceutical Sciences, Sun Yat-Sen University, Guangzhou 510006, China
}

Correspondence: Wei Li (liw@sysucc.org.cn) or Pei-qing Liu (liupq@mail.sysu.edu.cn)

These authors contributed equally: Yue-huai $\mathrm{Hu}$, Jie Liu

Received: 14 July 2019 Accepted: 9 January 2020

Published online: 1 April 2020 
(USA) and dissolved in dimethyl sulfoxide (DMSO) to $20 \mathrm{mM}$. Anisomycin (JNK activator, purity of $97.01 \%$ ) was obtained from Selleck Chemicals (USA) and diluted in DMSO to $50 \mathrm{mM}$. All the prepared regents were stored at $-20^{\circ} \mathrm{C}$.

Animals and treatments

The experimental animal procedures were approved by the Research Ethics Committee of Sun Yat-sen University and were conducted in accordance with the Guide for the Care and Use of Laboratory Animals (NIH Publication No. 85-23, revised 1996). Sprague-Dawley (SD) rats (males weighing 220-250 g, SPF grade, certification no. 44008500018304) were obtained from the Experimental Animal Center of Sun Yat-sen University (Guangzhou, China). The animals were randomly separated into two groups. Rats in the Dox group were intraperitoneally injected with Dox for a cumulative dose of $15 \mathrm{mg} / \mathrm{kg}$ Dox (5 mg/kg/injection at days 1,5 , and 9). The rats in the control group received an equal volume of normal saline (NS) in parallel. Each group had six animals.

Cell culture and treatment

$\mathrm{H} 9 \mathrm{c} 2$ cells were plated at a density of $1 \times 10^{6}$ cells per dish $(35 \mathrm{~mm})$ filled with Dulbecco's modified Eagle's medium (DMEM) (Gibco) a

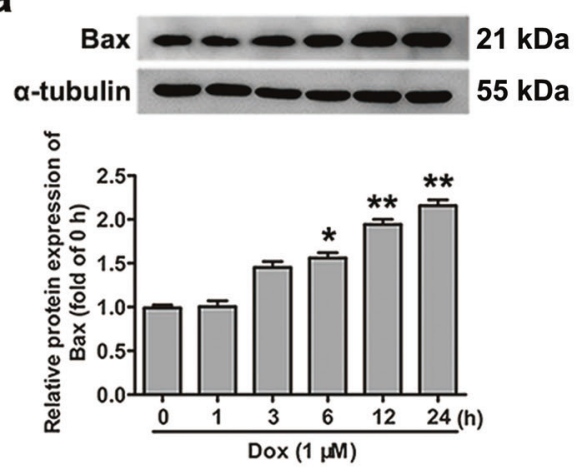

C

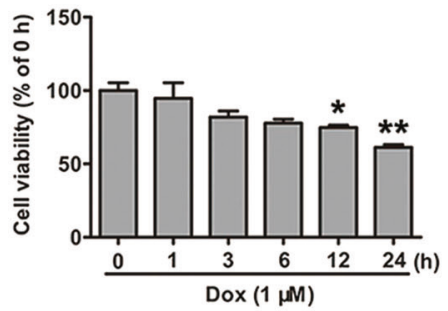

e
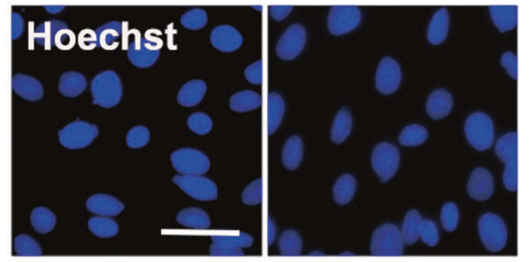

Con

Dox

g

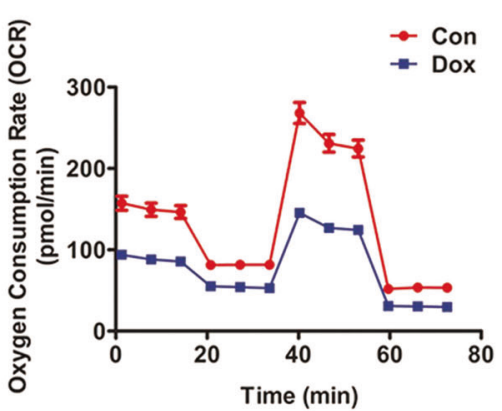

b

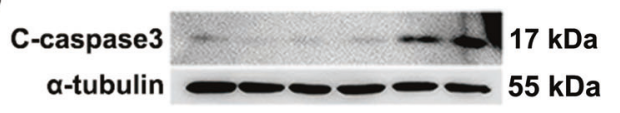

ธ

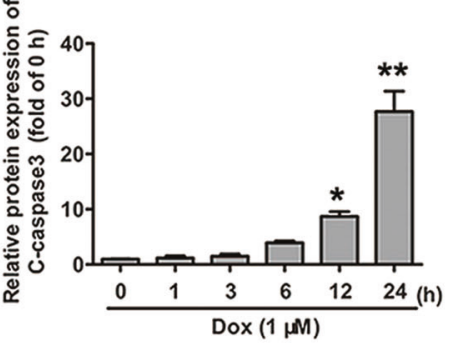

d

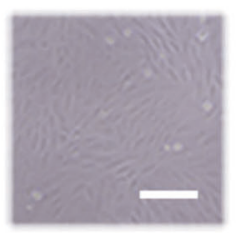

Con

f

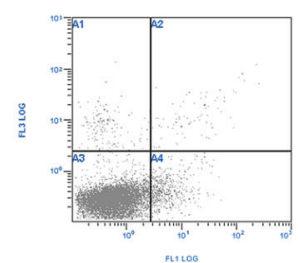

Con

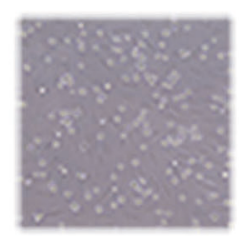

Dox

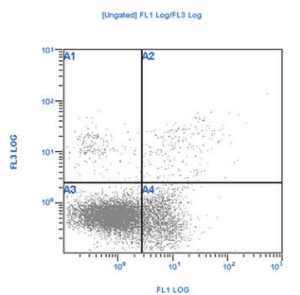

Dox h

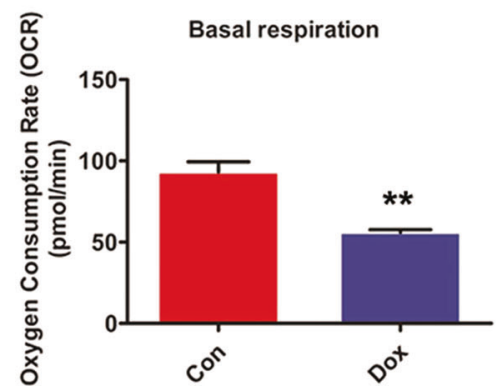

Fig. 1 Dox induces apoptosis and respiratory defects in H9c2 cells. H9c2 cells were incubated with $1 \mu \mathrm{M}$ Dox for the indicated time periods. a, $\mathbf{b}$ The expression of Bax and cleaved caspase- 3 was measured by Western blot analysis. c Cell viability was measured by the MTS assay. d The cellular morphology was observed by direct visualization with a light microscope. Scale bar: $200 \mu \mathrm{m}$. e Nuclear condensation and DNA fragmentation were observed in the Hoechst 33342-stained cells. Scale bar: $50 \mu \mathrm{m}$. $\mathbf{f}$ The apoptosis rate was detected by flow cytometry. $\mathbf{g}, \mathbf{h}$ Seahorse assay was used to detect the respiratory function of the H9c2 cells. The data are presented as the means \pm SEM. ${ }^{*} P<0.05$ and ${ }_{* *} P<0.01$ vs. the control group; $n=3$ 
supplemented with $10 \%$ fetal bovine serum. The cells were grown at $37^{\circ} \mathrm{C}$ in humidified $5 \% \mathrm{CO}_{2} . \mathrm{H} 9 \mathrm{c} 2$ cells were incubated with Dox $(1 \mu \mathrm{M})$ for the indicated times. The cells were incubated with SP600125 $(5 \mu \mathrm{M})$ and anisomycin $(50 \mu \mathrm{M}) 30 \mathrm{~min}$ before the Dox treatment. Adenovirus encoding SFRP1 was inoculated into the cells $12 \mathrm{~h}$ before the Dox treatment. CRISPR/Cas9-mediated knockdown of JNK (sgJNK) was carried out, and the following target sequence of sgRNA was used: 5'-CTCGGTAGGCTCGCTTAGCA-3'. H9c2 cells were transfected with vectors encoding Cas 9 and sgJNK. To upregulate the expression of JNK, we transfected the H9c2 cells with JNK plasmid by using Lipofectamine 2000 transfection reagent (Invitrogen Corporation) according to the manufacturer's protocol.

\section{Western blot analysis}

Western blot analysis was performed as previously described [16]. Primary antibodies against Bax (rabbit, diluted 1:1000), poly(ADPribose) polymerase 1 (PARP1) (rabbit, diluted 1:1000), caspase-3 (rabbit, diluted 1:1000), $\beta$-catenin (rabbit, diluted 1:1000), phosphorylated (P)-JNK (rabbit, diluted 1:1000), and total-JNK (T-JNK) (rabbit, diluted 1:1000) were purchased from Cell Signaling Technology. Antibodies against sFRP1 (rabbit, diluted 1:500) were obtained from Cloud-Clone Corp. (CCC, USA). Primary antibodies against $a$-tubulin (diluted 1:5000, Sigma, USA) and glyceraldehyde 3-phosphate dehydrogenase (diluted 1:1000, Sigma, USA) served as loading controls for whole-cell lysates. The band intensity was analyzed by Quantity One software (Bio-Rad, USA).

\section{Cell viability assay}

Cell viability was examined using the MTS assay. Briefly, H9c2 cells were cultured in 96-well plates. After treatment, the medium was replaced with $100 \mu \mathrm{L}$ of fresh medium containing $20 \mu \mathrm{L}$ of MTS reagent, and the cells were incubated in the dark for another $2 \mathrm{~h}$ at $37^{\circ} \mathrm{C}$. Then, the optical density of each well was measured at $490 \mathrm{~nm}$ by using a microplate reader (Tecan, Switzerland).

Determination of nuclear condensation and DNA fragmentation Nuclear condensation and DNA fragmentation were examined by using Hoechst 33342. Briefly, H9c2 cells were cultured in 96-well plates. After Dox treatment, $10 \mu \mathrm{g} / \mathrm{mL}$ Hoechst 33342 was added to the medium, and cells were incubated in the dark for another $10 \mathrm{~min}$ at $37^{\circ} \mathrm{C}$. Then, the cell culture medium was replaced with DMEM without phenol red, and the evidence of nuclear condensation and DNA fragmentation was photographed using an EVOS FL Auto Cell Imaging System (Life Technologies, Bothell, WA, USA).

\section{Flow cytometry assay}

The extent of cell apoptosis was assessed with an annexin V/propidium iodide (PI) apoptosis assay kit (BestBio, Shanghai, China). Briefly, $\mathrm{H} 9 \mathrm{c} 2$ cells were seeded in 6 -well plates and treated with Dox for $12 \mathrm{~h}$, harvested with trypsin without EDTA, and washed with PBS. Annexin V and PI staining was performed using an annexin V/PI apoptosis assay kit (BestBio, Shanghai, China) in accordance with the manufacturer's instructions. The stained cells were analyzed by flow cytometry (excitation at 488 $\mathrm{nm}$; emission at $530 \mathrm{~nm}$ ) with an EPICS XL instrument (Beckman Coulter, USA).

\section{Oxygen consumption rate}

The oxygen consumption rate (OCR) was measured using a Seahorse XFe96 Extracellular Analyzer (Agilent, CA) according to the manufacturer's instructions. The OCR was examined using the Seahorse XF Cell Mito Stress Test kit. H9c2 cells were incubated in minimum essential media (MEM) ( $\mathrm{pH}$ 7.4) supplemented with glucose $(10 \mathrm{mM})$ and pyruvate $(1 \mathrm{mM})$. After basal measurements were taken, oligomycin $(2 \mu \mathrm{M})$, carbonylcyanide $p$-trifluoromethoxyphenylhydrazone $(0.5 \mu \mathrm{M})$, and antimycin $\mathrm{A} /$ rotenone $(0.5 \mu \mathrm{M})$ were autoinjected into the experimental wells sequentially, and the respiration rate was measured three times followed by each injection. Each average basal or post-oligomycin treatment respiration rate was measured after the readings achieved a steady state.

\section{Quantitative reverse transcription PCR}

Quantitative reverse transcription PCR (RT-qPCR) was conducted as previously described [7]. Messenger RNA levels of target genes were measured by qRT-PCR, and $\beta$-actin served as an internal control.

The sequences of the rat-specific primers were as follows: Ptk 7, 5'-CATTGTCTGTCACCCATTCG-3' and 5'-GTTTGATAAGGAGGCTAC GG-3'; Daam 1, 5'-GCCCATGAGGTTTGTAAC-3' and 5'-AGCCAATGA GGGAGGTAT- $3^{\prime}$; and $\beta$-actin, $5^{\prime}$-TCGTGCGTGACATTAAAGAG- ${ }^{\prime}$ and 5'-ATTGCCGATAGTGATGACCT-3'.

\section{RESULTS}

Dox induces apoptosis and respiratory defects in H9c2 cells Dox is an effective anticancer anthracycline antibiotic with limited application because it induces acute and serious cardiotoxic side effects [3]. Oxidative stress and apoptosis are the most important mechanisms of Dox-induced cardiotoxicity [3]. In our study, H9c2 cells were treated with $1 \mu \mathrm{M}$ Dox for the indicated times. The protein expression of Bax and cleaved caspase- 3 were upregulated with increased Dox treatment times (Fig. 1a, b). The results from the MTS assay showed that cell viability was reduced as the Dox incubation time increased (Fig. 1c). Because cell injury was obvious at $12 \mathrm{~h}$, experimental measurements were taken after the cells were treated for $12 \mathrm{~h}$. Morphological damage induced by Dox was observed by light microscopy (Fig. 1d). Hoechst staining of the $\mathrm{H} 9 \mathrm{c} 2$ cells showed that Dox caused nuclear condensation and DNA fragmentation (Fig. 1e). A flow cytometer was used to a
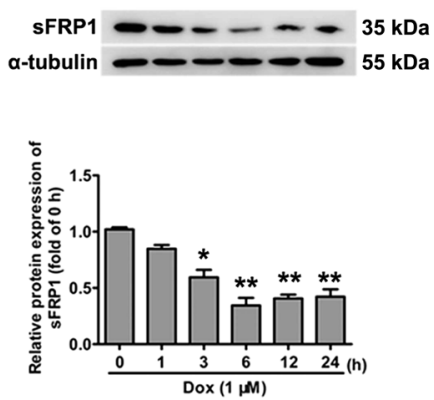

b
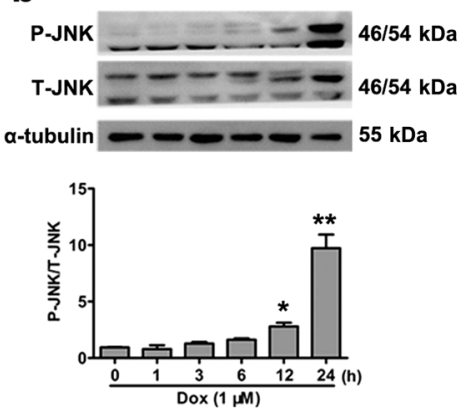

C
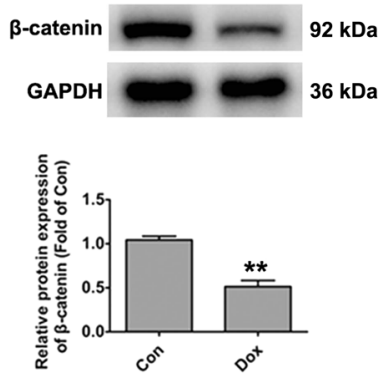

Fig. 2 Changes in SFRP1 and Wnt signaling in Dox-induced apoptosis. H9c2 cells were incubated with $1 \mu \mathrm{M}$ Dox for the indicated times. a The protein level of sFRP1 was measured by Western blot analysis. $\mathbf{b}$ The levels of P-JNK and T-JNK were detected by Western blot. $\mathbf{c}$ The level of $\beta$-catenin was detected by Western blot. The data are presented as the means \pm SEM. ${ }^{*} P<0.05$ and ${ }^{* *} P<0.01$ vs. the control group; $n=3$ 
a
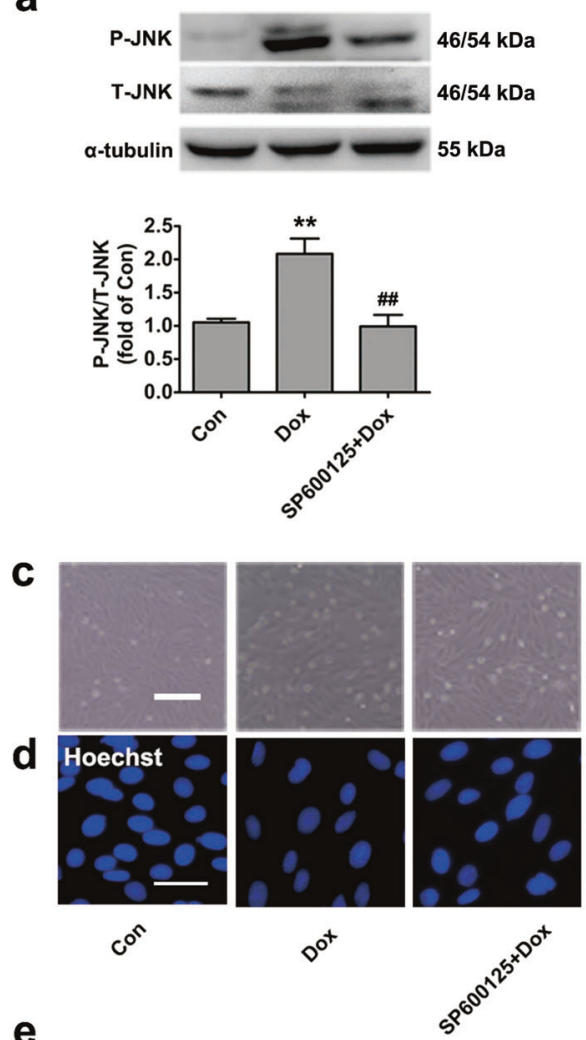

b

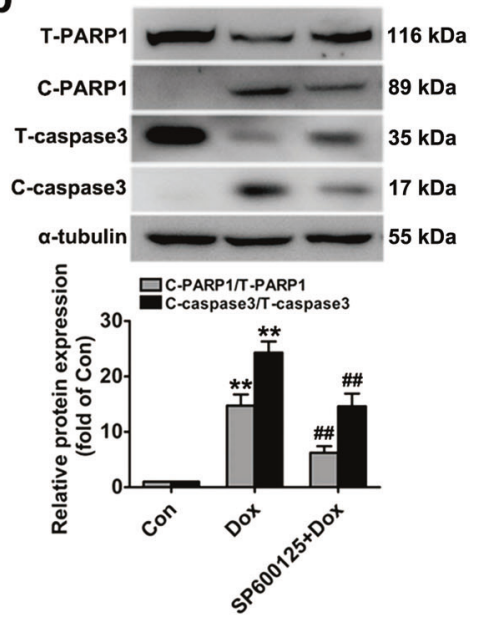

$\mathbf{f}$
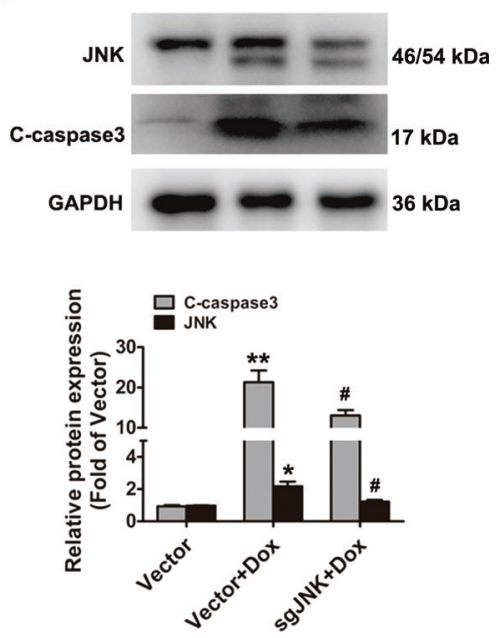

Fig. 3 Inhibition of Wnt/PCP-JNK signaling attenuates Dox-induced apoptosis. H9c2 cells were pretreated with SP600125 (5 $\mu$ M) for 30 min or were preinfected with sgJNK followed by Dox $(1 \mu \mathrm{M})$ incubation for $12 \mathrm{~h}$. a The levels of P-JNK and T-JNK were measured by Western blot analysis. b The expression of cleaved PARP1 and caspase-3 was determined by Western blot analysis. c Cellular morphology was observed by direct visualization with a light microscope. Scale bar: $200 \mu \mathrm{m}$. d Nuclear condensation and DNA fragmentation were observed in the Hoechst 33342-stained cells. Scale bar: $50 \mu \mathrm{m}$. e Cell viability was measured by the MTS assay. f The levels of JNK and cleaved caspase-3 were determined by Western blot analysis. The data are presented as the means \pm SEM. ${ }^{*} P<0.05$ and ${ }^{* *} P<0.01$ vs. the control or vector group. ${ }^{\#} P<0.05$ and ${ }^{\# \#} P<0.01$ vs. the Dox group or Vector + Dox group; $n=3$

evaluate the extent of $\mathrm{H} 9 \mathrm{c} 2$ cell apoptosis, and the results implied that Dox treatment increased both early and late apoptosis in the cells (Fig. 1f). In addition, results from the seahorse analysis showed serious respiratory defects in the $\mathrm{H} 9 \mathrm{c} 2$ cells. (Fig. 1g, h).

Changes in SFRP1 and Wnt signaling during Dox-induced apoptosis

In our previous study [7], the protein level of sFRP1 was reduced in Dox-treated neonatal rat cardiomyocytes (NRCMs). In this study, reduced sFRP1 levels were also detected in the Dox-treated H9c2 cells (Fig. 2a).

A recent study showed an association between the Wnt/PCPJNK pathway and apoptosis [17]. To explore the involvement of the Wnt/PCP-JNK pathway in Dox-induced apoptosis, H9c2 cells were incubated with Dox, and the levels of P-JNK and T-JNK were detected. The level of P-JNK and the ratio of P-JNK/T-JNK were increased after cells were incubated with Dox (Fig. 2b). In addition, canonical Wnt/ $\beta$-catenin signaling was suppressed by Dox, as shown by the protein expression of $\beta$-catenin (Fig. $2 c$ ).
Inhibition of Wnt/PCP-JNK signaling attenuates Dox-induced apoptosis

To further explore the role of Wnt/PCP-JNK signaling in Doxinduced apoptosis, H9c2 cells were pretreated with SP600125 (5 $\mu M)$, an inhibitor of the Wnt/PCP-JNK pathway, for $30 \mathrm{~min}$ followed by incubation with Dox. It was obvious that the upregulated Wnt/PCP-JNK signaling caused by Dox was suppressed by SP600125 (Fig. 3a). In addition, the inhibition of Wnt/ PCP-JNK signaling led to the reduced levels of cleaved PARP1 and caspase-3 (Fig. 3b). Dox-induced morphological damage was diminished by SP600125, as observed in cells with a light microscope (Fig. 3c). Nuclear condensation and DNA fragmentation were ameliorated by SP600125, as revealed by Hoechst 33342 staining (Fig. 3d). The results from the MTS assay showed that the reduced cell viability caused by Dox was reversed by SP600125 (Fig. 3e). In addition, the expression of cleaved caspase3 that had been induced by Dox was reduced by CRISPR/Cas9 system-mediated knockdown of JNK (Fig. 3f). The results described above indicated that the Wnt/PCP-JNK signaling pathway was involved in Dox-induced apoptosis of H9c2 cells. 

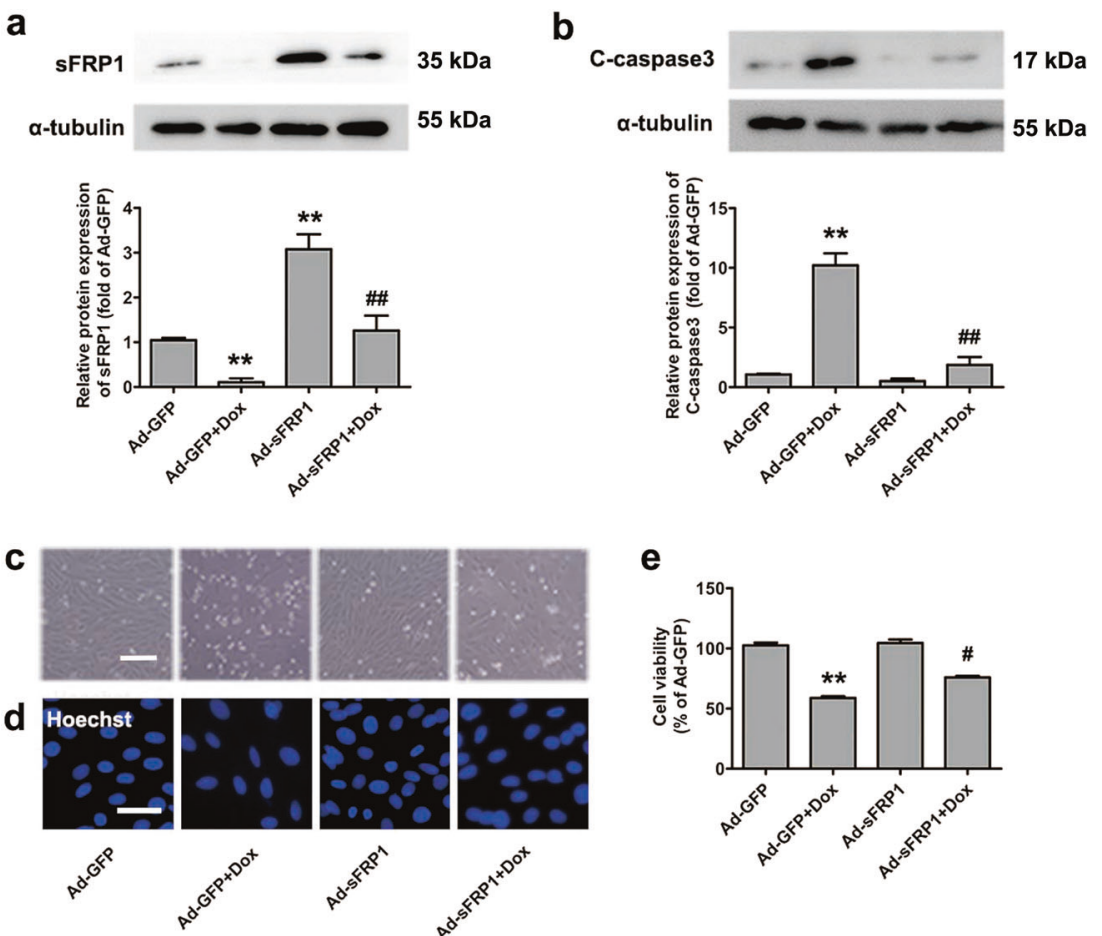

Fig. 4 sFRP1 protects H9c2 cells from Dox-induced apoptosis. H9c2 cells were preinfected with Ad-sFRP1 followed by Dox (1 $\mu \mathrm{M})$ incubation for $12 \mathrm{~h}$. a The level of sFRP1 was determined by Western blot analysis. $\mathbf{b}$ The expression of cleaved caspase-3 was determined by Western blot analysis. c Cellular morphology was observed by direct visualization with a light microscope. Scale bar: $200 \mu \mathrm{m}$. d Nuclear condensation and DNA fragmentation were observed in the Hoechst 33342-stained cells. Scale bar: $50 \mu \mathrm{m}$. e Cell viability was measured by the MTS assay. The data are presented as the means \pm SEM. ${ }^{* *} P<0.01$ vs. the Ad-GFP group; ${ }^{\#} P<0.05$ and ${ }^{\# \#} P<0.01$ vs. the Ad-GFP + Dox group; $n=3$

sFRP1 protects H9c2 cells from Dox-induced apoptosis

The results described above showed that Dox caused a reduction in sFRP1 level; therefore, a recombinant adenovirus vector encoding SFRP1 (Ad-sFRP1) was used to investigate the effect of sFRP1 on Dox-induced apoptosis. The results showed that overexpression of sFRP1 was successfully induced by the AdsFRP1 vector (Fig. 4a). In addition, the results from the Western blot analysis showed that the increased expression of cleaved caspase-3 induced by Dox was suppressed by the overexpression of sFRP1 (Fig. 4b). The Dox-induced morphological damage and nuclear condensation were diminished by Ad-sFRP1 (Fig. 4c, d). The results from the MTS assay showed that the reduced cell viability caused by Dox was reversed by the overexpression of sFRP1 (Fig. 4e). The results described above indicated that sFRP1 played a protective role in the Dox-induced apoptosis of $\mathrm{H} 9 \mathrm{c} 2$ cells.

Upregulation of sFRP1 inhibits the Wnt/PCP-JNK signaling activity sFRP1 is reportedly the endogenous inhibitor of both the canonical Wnt/B-catenin and noncanonical Wnt/PCP-JNK pathways [15]. In our previous study, we explored the regulatory relationship between sFRP1 and Wnt/ $\beta$-catenin in Dox-induced cardiomyopathy [7]. In this study, we focused on the regulatory relationship between SFRP1 and Wnt/PCP-JNK pathway factors in Dox-induced apoptosis of $\mathrm{H} 9 \mathrm{c} 2$ cells. The RT-qPCR results showed that Dox promoted the transcription of Ptk 7 and Daam 1, two Wnt/PCP-JNK pathway target genes, but this effect was inhibited by the overexpression of SFRP1 (Fig. 5a, b). In addition, the upregulated P-JNK expressed induced by Dox was suppressed by sFRP1 (Fig. 5c). Anisomycin is an agonist of the JNK pathway. In our experiment, we found that the upregulated levels of P-JNK and cleaved caspase- 3 that had been induced by Dox were downregulated by the overexpression of sFRP1, but these effects were diminished by anisomycin $(50 \mu \mathrm{M})$ treatment (Fig. $5 \mathrm{~d}$ ). Overexpression of JNK exhibited a similar effect as that of anisomycin (Fig. 5e). In addition, anisomycin treatment also induced a significant increase in P-JNK and in cleaved PARP1 and caspase-3, but these effects were prevented in cells preinfected with Ad-sFRP1 (Fig. $5 f, g$ ). The results described above indicated that SFRP1 protected H9c2 cells from Dox-induced apoptosis by inhibiting the Wnt/PCP-JNK pathway.

Changes to the canonical and noncanonical Wnt signaling pathways by Dox-induced injury in vivo

To further identify the changes in Wnt signaling upon Dox treatment, SD rats were intraperitoneally injected with a cumulative dose of $15 \mathrm{mg} / \mathrm{kg}$ Dox $(5 \mathrm{mg} / \mathrm{kg} /$ injection at days 1 , 5 , and 9), and the control rats received the same volume of NS in parallel. Echocardiography results revealed a decrease in the ejection fraction, fractional shortening, and cardiac output (CO) (Fig. 6a-d). The hearts of the Dox-treated rats were notably smaller than those of the control group (Fig. 6e). Dox treatment also increased the disorganization of the myocytes and the apoptosis rate, as revealed by the hematoxylin- and eosin-stained cells (Fig. 6f) and TUNEL (terminal deoxynucleotidyl transferase dUTP nick end labeling) assay results (Fig. 6g). Following Dox treatment, the protein expression of P-JNK was increased (Fig. 6h), while the protein level of $\beta$-catenin was decreased (Fig. 6i). These results indicated that the canonical Wnt/ $\beta$-catenin pathway was inhibited and that the noncanonical Wnt/PCP-JNK pathway was activated upon Dox treatment.

\section{DISCUSSION}

Although Dox is one of the most effective chemotherapeutic drugs for treating many cancers, the lethal nature of Dox-induced 
a

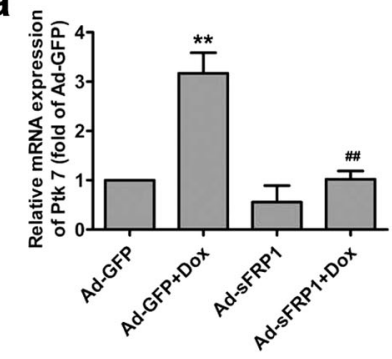

b

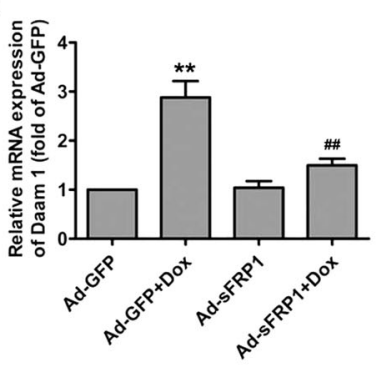

C
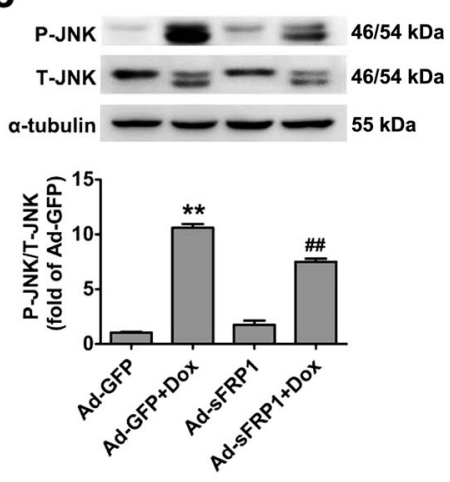

d
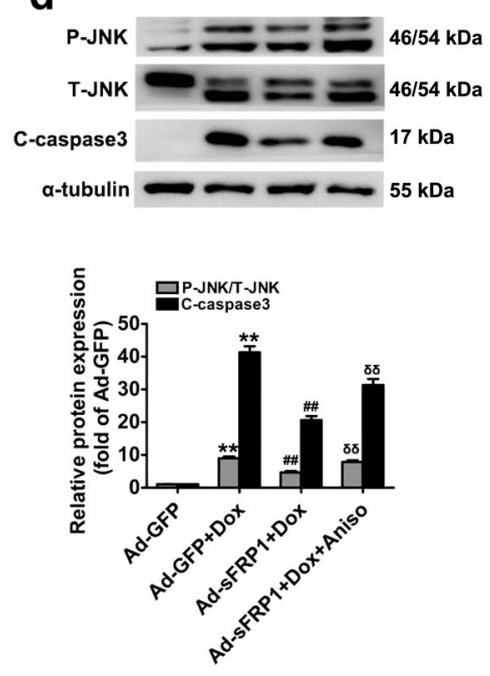

f
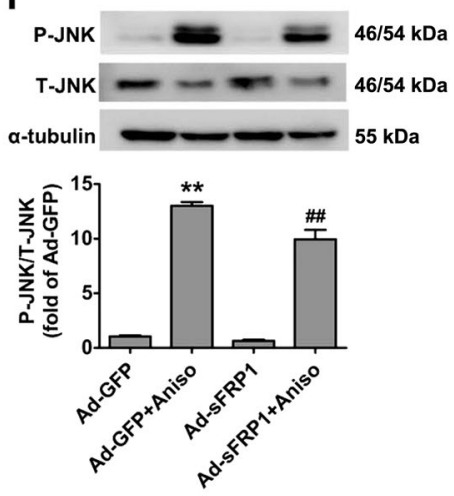

e
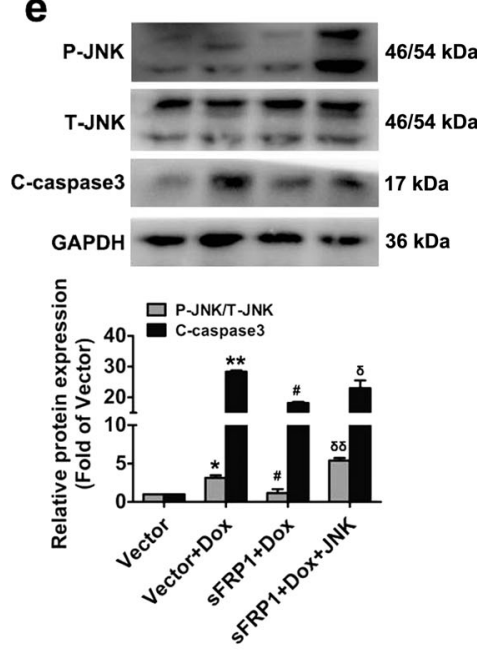

g

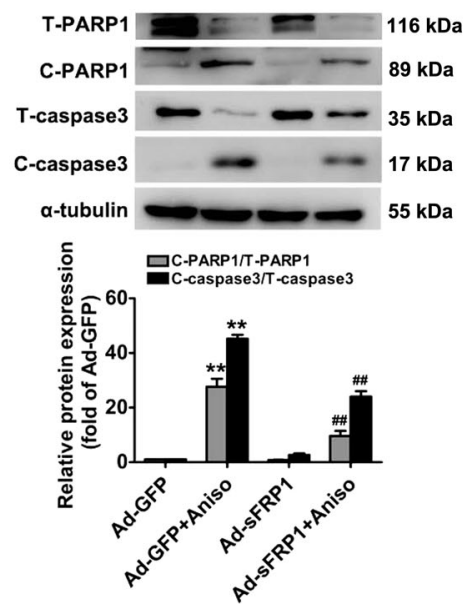

Fig. 5 Upregulation of sFRP1 inhibits Wnt/PCP-JNK signaling activity. H9c2 cells were preinfected with Ad-sFRP1 followed by incubation with Dox $(1 \mu \mathrm{M})$, and anisomycin (Aniso) $(50 \mu \mathrm{M})$ or a JNK plasmid was used to activate the Wnt/PCP-JNK pathway. a, b Messenger RNA (mRNA) expression of the Wnt/PCP-JNK target genes. c, $\mathbf{f}$ The levels of P-JNK, T-JNK and cleaved caspase-3 were determined by Western blot analysis. d, e The levels of P-JNK, T-JNK and cleaved caspase-3 were determined by Western blot analysis. $\mathbf{g}$ The expression of cleaved PARP1 and caspase- 3 was measured by Western blot analysis. The data are presented as the means \pm SEM. ${ }^{*} P<0.05$ and ${ }^{* *} P<0.01$ vs. the Ad-GFP or vector group. ${ }^{\# \#} P<0.01$ vs. the Ad-GFP + Dox group or Ad-GFP + Aniso group. ${ }^{\delta} p<0.05,{ }^{\delta} \delta<0.01$ vs. the Ad-sFRP1 + Dox group; $n=3$

cardiotoxicity is a serious problem. To date, strategies using pharmaceutical agents to prevent or reduce Dox cardiotoxicity have been tested in many studies. Dexrazoxane is the only compound to protect against Dox-induced cardiotoxicity [18]. Diuretics, a low salt diet, $\beta$-blockers [19-21], and angiotensinconverting enzyme inhibitors [22] are used in the clinic to relieve symptoms. However, to date, no single drug has been able to completely prevent Dox-induced cardiotoxicity clinically. A better understanding of the cardiotoxic mechanism of Dox may lead to the development of new therapeutic strategies. It is of great clinical significance to search for effective therapeutic targets.

In our study, we demonstrated the important role of SFRP1 in Dox-induced cardiotoxicity. We found that the level of sFRP1 was decreased in both the NRCMs [7] and H9c2 cells (Fig. 2a). Ectopic expression of sFRP1 protected H9c2 cells from Dox-induced apoptosis. In this study, we also studied the mechanism by which sFRP1 effectively protects cells from Dox-induced apoptosis. Numerous studies have shown that Dox induces apoptosis through various pathways [23]. It is thought that mitochondria play a significant role in Dox-induced apoptosis by regulating the release of cytochrome $c$, which results in the activation of caspases and subsequent cell death $[3,24]$. Several previous studies have suggested that SFRP1 plays an antiapoptotic role in cells exposed to ceramide [25], hypoxia [26], and/or inflammation [27]. In these studies, the expression of cleaved caspase- 3 was suppressed by sFRP1. In our study, we obtained results consistent with the results of these previous studies.

The Wnt signaling network comprises several signaling pathways, of which the best studied are the canonical Wnt/ $\beta$-catenin pathway and noncanonical Wnt/PCP-JNK pathway [28]. These two pathways are primarily thought to act in a mutually repressive manner because they compete for common proteins, such as the scaffolding protein disheveled [29]. Therefore, it is reasonable that as the level of Wnt/PCP-JNK signaling is upregulated, the level of Wnt/ $\beta$-catenin signaling is downregulated after Dox treatment. Our research showed that the inhibition of Wnt/PCP-JNK signaling contributed to the protective effect of sFRP1. In addition, anisomycin treatment induced the activation of Wnt/PCP-JNK signaling and the apoptosis of the $\mathrm{H} 9 \mathrm{c} 2$ cells, and these effects were suppressed by SFRP1. The results described above led to the conclusion that sFRP1 protected the H9c2 cells from Dox-induced apoptosis by inhibiting Wnt/PCP-JNK signaling. In addition, in our 
a

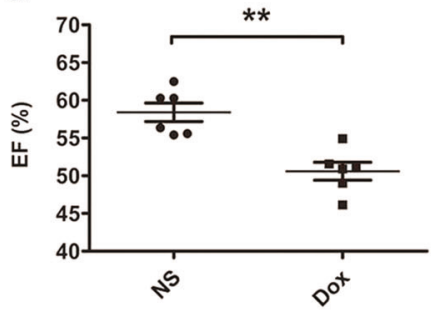

b

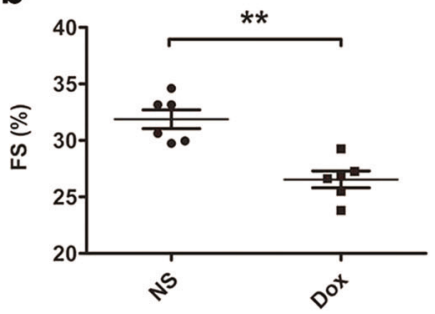

C

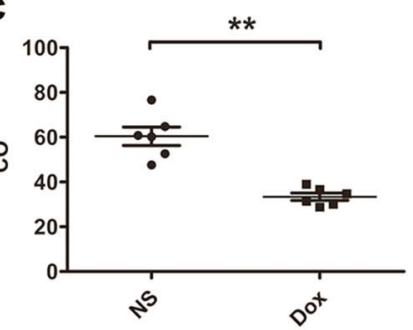

d

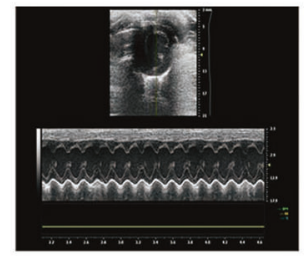

e

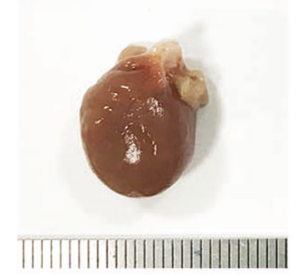

f

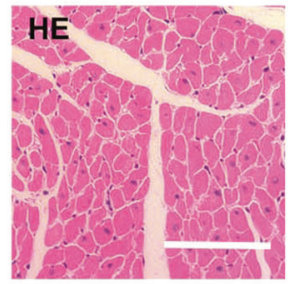

NS
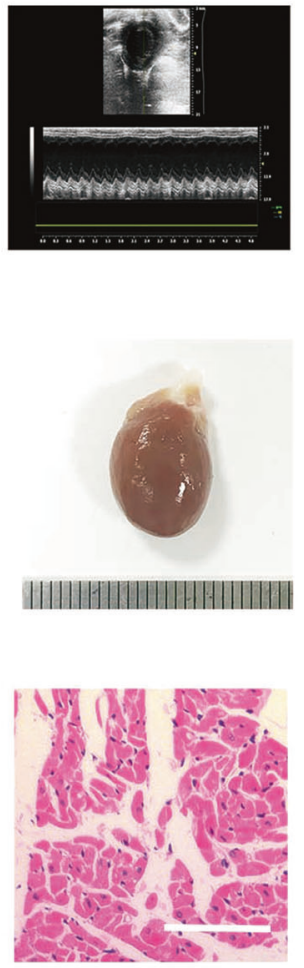

Dox

g
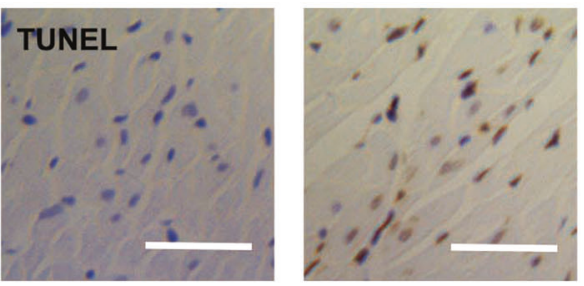

h


i

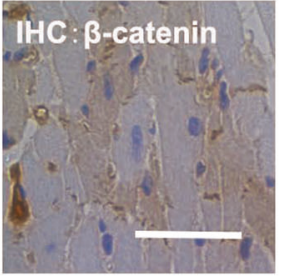

NS

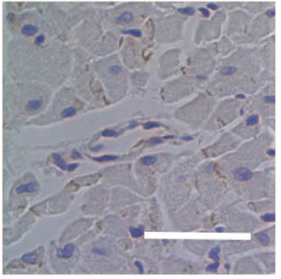

Dox

Fig. 6 Changes in the canonical and noncanonical Wnt signaling pathways in cells with Dox-induced injury in vivo. SD rats were intraperitoneally injected with Dox (cumulative dose was $15 \mathrm{mg} / \mathrm{kg}$ ) or sterile NS. a-c The echocardiographic parameters of ejection fraction (EF), fractional shortening (FS), and cardiac output (CO) were detected. d-f Echocardiogram of the gross heart and images of an HE-stained transection of the left ventricle are shown. Scale bar: $50 \mu \mathrm{m}$. g Apoptosis of rat heart cells was detected by TUNEL staining. Scale bar: $50 \mu \mathrm{m}$. $\mathbf{h}, \mathbf{i}$ The expression of P-JNK and $\beta$-catenin was examined by immunofluorescence and immunohistochemistry, respectively. Scale bar: $50 \mu \mathrm{m}$. Representative images of five independent experiments are presented. The data are presented as the means \pm SEM. ${ }^{* *} P<0.01$ vs. the NS group; $n=6$

previous study [7], we found that sFRP1 might regulate Doxinduced apoptosis by interacting with other proteins, including those not in the Wnt pathway. For example, it could attenuate apoptosis by inhibiting the activity of PARP1 (which can be activated as a DNA damage receptor after DNA-damaged fragments are identified) in the nucleus. Thus, it is believed that the protective effect of sFRP1 against Dox-induced apoptosis is a comprehensive result, but more research is needed to reveal it completely.

\section{ACKNOWLEDGEMENTS}

This research was supported by grants from the National Natural Science Foundation of China (81872860, 81803521, 81673433, and 81572925), Local Innovative and Research Teams Project of Guangdong Pearl River Talents Program (2017BT01Y093), National Major Special Projects for the Creation and Manufacture of New Drugs (2019ZX09301104), Special Program for Applied Science and Technology of Guangdong Province (2015B020232009), National Engineering and Technology Research Center for New Druggability Evaluation (Seed Program of Guangdong
Province, 2017B090903004), Guangzhou Science and Technology Program Project (201604020121), and YangFan Project of Guangdong Province (2014YT02S044).

\section{AUTHOR CONTRIBUTIONS}

YHH designed and performed the research; Jie Liu, Jing Lu, and FHH wrote the paper; PXW analyzed the data; JXC and YG prepared the reagents; and JJW, WL, and PQL designed the research.

\section{ADDITIONAL INFORMATION}

Competing interests: The authors declare no competing interests.

\section{REFERENCES}

1. Razavi-Azarkhiavi K, Jaafari MR, Abnous K, Razavi BM, Jafarian AH, Hassani FV. et al. The cardiotoxic mechanism of doxorubicin (DOX) and pegylated liposomal DOX in mice bearing C-26 colon carcinoma: a study focused on microRNA role for toxicity assessment of new formulations. Pharmaol Res. 2017;34:1849-56. 
sFRP1 protects H9c2 cardiac myoblasts from doxorubicin-induced apoptosis $\mathrm{YH}$ Hu et al.

2. Bruynzeel AM, Abou El Hassan MA, Schalkwijk C, Berkhof J, Bast A, Niessen HW, et al. Anti-inflammatory agents and monoHER protect against DOX-induced cardiotoxicity and accumulation of CML in mice. Br J Cancer. 2007;96:937-43.

3. Shi Y, Moon M, Dawood S, McManus B, Liu PP. Mechanisms and management of doxorubicin cardiotoxicity. Herz. 2011;36:296-305.

4. Bruynzeel AM, Vormer-Bonne S, Bast A, Niessen HW, van der Vijgh WJ. Long-term effects of 7-monohydroxyethylrutoside (monoHER) on DOX-induced cardiotoxicity in mice. Cancer Chemother Pharmacol. 2007;60:509-14.

5. Mohammed MK, Shao C, Wang J, Wei Q, Wang X, Collier Z, et al. Wnt/beta-catenin signaling plays an ever-expanding role in stem cell self-renewal, tumorigenesis and cancer chemoresistance. Genes Dis. 2016:3:11-40.

6. Korkut C, Ataman B, Ramachandran P, Ashley J, Barria R, Gherbesi N, et al. Transsynaptic transmission of vesicular Wnt signals through Evi/Wntless. Cell. 2009;139:393-404.

7. Hu Y, Guo Z, Lu J, Wang P, Sun S, Zhang $Y$, et al. sFRP1 has a biphasic effect on doxorubicin-induced cardiotoxicity in a cellular location-dependent manner in NRCMs and Rats. Arch Toxicol. 2019;93:533-46.

8. Matsuyama M, Nomori A, Nakakuni K, Shimono A, Fukushima M. Secreted Frizzled-related protein 1 (Sfrp1) regulates the progression of renal fibrosis in a mouse model of obstructive nephropathy. J Biol Chem. 2014;289:31526-33.

9. Asad M, Wong MK, Tan TZ, Choolani M, Low J, Mori S, et al. FZD7 drives in vitro aggressiveness in Stem-A subtype of ovarian cancer via regulation of noncanonical Wnt/PCP pathway. Cell Death Dis. 2014;5:e1346.

10. Lopez-Escobar B, Cano DA, Rojas A, de Felipe B, Palma F, Sanchez-Alcazar JA, et al. The effect of maternal diabetes on the Wnt-PCP pathway during embryogenesis as reflected in the developing mouse eye. Dis Model Mech. 2015;8:157-68.

11. Dufourcq P, Couffinhal T, Ezan J, Barandon L, Moreau C, Daret D, et al. FrzA, a secreted frizzled related protein, induced angiogenic response. Circulation. 2002; 106:3097-103.

12. Jones SE, Jomary C. Secreted Frizzled-related proteins: searching for relationships and patterns. BioEssays. 2002;24:811-20.

13. Sklepkiewicz P, Shiomi T, Kaur R, Sun J, Kwon S, Mercer B, et al. Loss of secreted frizzled-related protein-1 leads to deterioration of cardiac function in mice and plays a role in human cardiomyopathy. Circ Heart Fail. 2015;8:362-72.

14. Matsuyama M, Aizawa S, Shimono A. Sfrp controls apicobasal polarity and oriented cell division in developing gut epithelium. PLoS Genet. 2009;5: e1000427.

15. Satoh W, Matsuyama M, Takemura H, Aizawa S, Shimono A. Sfrp1, Sfrp2, and Sfrp5 regulate the Wnt/beta-catenin and the planar cell polarity pathways during early trunk formation in mouse. Genesis. 2008;46:92-103.
16. Guo Z, Lu J, Li J, Wang P, Li Z, Zhong Y, et al. JMJD3 inhibition protects against isoproterenol-induced cardiac hypertrophy by suppressing beta-MHC expression. Mol Cell Endocrinol. 2018;477:1-14.

17. Zhang $\mathrm{Q}$, Liu $\mathrm{Y}$, Wang $\mathrm{H}, \mathrm{Ma} \mathrm{L}, \mathrm{Xia} \mathrm{H}, \mathrm{Niu}$ J, et al. The preventive effects of taurine on neural tube defects through the Wnt/PCP-Jnk-dependent pathway. Amino Acids. 2017;49:1633-40.

18. American Society of Clinical Oncology 2008 Clinical practice guideline update summary: use of chemotherapy and radiation therapy protectants. J Oncol Pract. 2008;4:277-9.

19. Malcom J, Arnold O, Howlett JG, Ducharme A, Ezekowitz JA, Gardner M, et al Canadian Cardiovascular Society Consensus Conference guidelines on heart failure-2008 update: best practices for the transition of care of heart failure patients, and the recognition, investigation and treatment of cardiomyopathies. Can J Cardiol. 2008;24:21-40.

20. Shaddy RE, Olsen SL, Bristow MR, Taylor DO, Bullock EA, Tani LY, et al. Efficacy and safety of metoprolol in the treatment of doxorubicin-induced cardiomyopathy in pediatric patients. Am Heart J. 1995;129:197-9.

21. Spallarossa P, Garibaldi S, Altieri P, Fabbi P, Manca V, Nasti S, et al. Carvedilo prevents doxorubicin-induced free radical release and apoptosis in cardiomyocytes in vitro. J Mol Cell Cardiol. 2004;37:837-46.

22. Sacco G, Mario B, Lopez G, Evangelista S, Manzini S, Maggi CA. ACE inhibition and protection from doxorubicin-induced cardiotoxicity in the rat. Vasc Pharmacol. 2009;50:166-70.

23. Songbo $M$, Lang $H$, Xinyong $C$, Bin $X$, Ping $Z$, Liang S. Oxidative stress injury in doxorubicin-induced cardiotoxicity. Toxicol Lett. 2019;307:41-8.

24. Green PS, Leeuwenburgh C. Mitochondrial dysfunction is an early indicator of doxorubicin-induced apoptosis. Biochim Biophys Acta. 2002;1588:94-101.

25. Han X, Amar S. Secreted frizzled-related protein 1 (SFRP1) protects fibroblasts from ceramide-induced apoptosis. J Biol Chem. 2004;279:2832-40.

26. Tao J, Abudoukelimu M, Ma YT, Yang YN, Li XM, Chen BD, et al. Secreted frizzled related protein 1 protects $\mathrm{H} 9 \mathrm{C} 2$ cells from hypoxia/re-oxygenation injury by blocking the Wnt signaling pathway. Lipids Health Dis. 2016;15:72.

27. Barandon L, Casassus F, Leroux L, Moreau C, Allieres C, Lamaziere JM, et al. Secreted frizzled-related protein-1 improves postinfarction scar formation through a modulation of inflammatory response. Arterioscler Thromb Vasc Biol. 2011;31:e80-7.

28. Routledge $D$, Scholpp S. Mechanisms of intercellular Wnt transport. Development. 2019;146:dev176073.

29. Gao C, Chen YG. Dishevelled: the hub of Wnt signaling. Cell Signal. 2010;22 717-27. 